\title{
Mga leyendang Chabacano: Salamin sa pananampalatayang Zamboangueño
}

Reyes, Aubrey $₫$

Western Mindanao State University, Philippines (aubreyfreyes80@gmail.com)

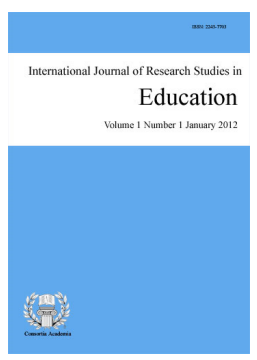

Accepted: 25 October 2020

ISSN: 2243-7703 Online ISSN: 2243-7711

OPEN ACCESS

\section{Abstract}

This study aims to identify the factors that influence the Zamboangueño's faith reflected in the Chabacano legends of Zamboanga city. Historical and sociological perspectives were used to determine the narrative of their faith. The legends published in the literary book by Orlando B. Cuartocruz entitled Zamboanga Chabacano Folk Literature (1990) and Dr. Teresita P. Semorlan Mga Leyendang Chabacano (1984) are the data of this study. The method of faith was examined and found to have factors that influenced it. The first is environmental forces such as the fall of the coconut fruit in front of the cat, the spread of cholera in the village and the survival of the people against such diseases, the loss of the crops due to drought, famine in the village, illness and death of people because of cholera, and the exaltation of the Virgin, the appearance of the image of the Saint, the occurrence of severe female disease and death. The second is human forces such as the invasion of bandit groups, the existence of a religious festival, the religiosity of the Moros, the celebration of the first centenary of the village of Tetuan, the celebration of the day of the Patron of the village, faith in the saint, the influenced of the catholic friend of the princess, the village survives against the Moro invaders, and Graciano's accident and death. Based on the findings, Christianity and Islam have a huge impact and influenced in the spiritual aspects and faith of the Zamboangueños.

Keywords: leyenda; Chabacano; salik; pananampalataya; Zamboangueňo 


\section{Mga leyendang Chabacano: Salamin sa pananampalatayang Zamboangueño}

\section{Introduksyon}

Maituturing na paganismo o paniniwalang pagano ang pananampalataya ng mga sinaunang ninuno. May kaugnayan ang ganitong uri ng pananampalataya sa pagsamba sa kalikasan o mga bagay sa kalikasan gaya ng araw, bituin, puno hayop at iba pa. Naniniwala ang mga ninuno na ang pinakamakapangyarihang Diyos ay si Bathala. Bukod kay Bathala ay sumasamba rin sila sa mga anito. Nag-aalay sila ng mga panalangin at pagkain upang mapagbigyan ang kanilang mga kahilingan at magkaroon ng masaganang buhay.

Hindi maikakailang ang Kristiyanismo ang pinakamalaking impluwensiya ng mga Espanyol sa ating mga Pilipino. Sa Asya, tanging Pilipinas lamang ang kinikilalang Kristiyanong bansa. Ang matandang paniniwala ng mga Pilipino noon ay napalitan ng bagong paniniwalang itinuro ng mga misyonerong Espanyol. Ang mga Pilipino noon at tinuruan nilang magdasal, at magsimba, at magbasa ng Bibliya - banal na aklat ng mga Katoliko. Madali namang tinanggap ng maraming katutubo ang mga ito dahil sa ilang matandang paniniwala sa kababalaghan ng mga katutubong imahen tulad ng mga anito at iba pa (del Castillo, 2016; del Castillo, 2015).

Laganap naman sa bahaging Mindanao ang Islam o relihiyon ng mga Muslim. Pamana ito ng mga Arabe sa ating bansa. Ito ay isang uri ng paniniwalang monoteismo na nangangahulugang "pagsuko sa kagustuhan ng Diyos na si Allah". Totoong dahil sa Kristiyanismo ay nagbago ang pagpapahalaga at ugali ng mga Pilipino. Dahil sa pagiging likas na maka-Diyos at madasalin ng mga Pilipino ay madali nilang natanggap at naisabuhay ang maraming mga paniniwala at kaugaliang Espanyol na karamihan ay nakaugat sa Kristiyanismo. Bagamat marami ang yumakap sa pananampalatayang ito, nanatiling tapat naman sa kanilang relihiyon ang mga Pilipinong Muslim sa bahaging Mindanao. Hindi nila pinakinggan ang mga paring misyonero at nanindigan silang walang ibang Diyos para sa kanila kundi si Allah. Ito rin ang naging dahilan kung bakit hindi nasakop ng mga Espanyol ang malaking bahagi ng Mindanao (Julian at Lontoc, 2016).

Isang lungsod ang Zamboanga na may matinding pananampalataya, mapa-Kristiyano man o Muslim. Makikita ito sa kanilang lugar na binubuo ng napakaraming simbahan/masjid. Nariyan din ang napakaraming deboto ng Birhen del Pilar na nagsisindi ng kandila, nagdiriwang ng mga pista, nag-aayuno at marami pang iba. Bukod sa pananampalataya may mayaman ding kultura ang mga Zamboangueño.

Sinasabing walang may-akda ang tradisyunal o salindilang literatura, subalit alam ng tao na ito ay gawa ng ating mga ninuno. Produkto ito (materyal at di-materyal) ng katalinuhan at kaalaman ng mga matatanda. Tinatawag din ang buhay na buhay na mga panitikang ito na folklore, at isa sa mga ito ay ang alamat. Ang mga alamat ay mga salaysayin ng kahanga-hangang pangyayaring nagpasalin-salin mula sa mga naunang henerasyon at pinaniniwalaan sa dahilang may makasaysayan itong batayan. Itinuturing na makasaysayan ang diwa nito, nauukol sa isang bagay, pook, at pangyayaring may kabuluhang historikal. Pinakalayunin nito ang gunitain ang mga pangyayaring may kinalaman sa lumipas na panahon. Nagaganap ang alamat sa isang tiyak na lugar at pinag-uugnay nito ang nakaraan at ngayon. Ang bawat rehiyon sa Pilipinas ay may maipagmamalaking mga alamat na dapat pag-aaralan. May maipagmamalaki ring alamat ang lungsod Zamboanga na kilala sa tawag na leyenda. Marami sa mga leyenda ng lungsod ng Zamboanga ang nagsasalaysay tungkol sa pananampalataya. Inalam ng mananaliksik kung bakit ganito ang karamihan sa kanilang tema, ang implikasyon nito sa mga buhay ng mga Zamboangueño, at kung ano ang mga salik na nakaimpluwensiya sa pananampalataya ng mga zamboangueňo na masasalamin sa mga leyendang chabacano ng lungsod Zamboanga.

\section{Metodolohiya}

Isang disenyong kwalitatibo ang pag-aaral na ito. Ginamit ang dokumentaryong pamamaraan ng pagsusuri. Ang datos na ginamit ng mananaliksik ay ang mga leyenda ng lungsod Zamboanga na nailathala na sa mga aklat 
Mga leyendang Chabacano: Salamin sa pananampalatayang Zamboangueño

panliteratura nina Orlando B. Cuartocruz na pinamagatang "Zamboanga Chabacano Folk Literature" (1990) at aklat ni Teresita P. Semorlan "Mga Leyendang Chabacano" (1984). Labinlima (15) ang kabuuan ng leyendang sinuri batay sa tema ng pananampalataya sa Panginoon. . Ang mga leyendang ito ay inalisa gamit ang teoryang historikal at sosyolohikal. Mula sa mga leyendang ito, ay isinagawa ang desrkriptibong pagsusuri sa mga salik na nakaimpluwensiya sa iba't ibang paraan ng pananampalataya ng mga Zamboangueño batay sa impluwensiyang pangkalikasan at pantao. Upang makakuha ang mananaliksik ng pagpapakahulugan, interpretasyon at kaalaman ng mga Zamboangueño tungkol sa kanilang pananampalataya at paraan ng pananampalataya. Nagsagawa ng pakikipanayam ang mananaliksik mula sa iba't ibang taal na Zamboangueño.

\section{Paglalahad ng mga resulta}

Ang seksyon na ito ay naglalahad at nagsusuri ng mga datos upang maitatag ang mga salik na nakaimpluwensya sa Paraan ng Pananampalatayang Zamboangueño: (a) Puwersang pangkalikasan (b) Puwersang pantao. Makikita sa kasunod na pahina ang Talahanayan ng mga salik na nakaimpluwensiya sa pananampalatayang Zamboangueño (a) puwersang pangkalikasan (b) puwersang pantao.

\section{Talahanayan 1}

Mga salik na nakaimpluwensiya sa paraan pananampalataya ng mga Zamboangueño

\begin{tabular}{|c|c|c|c|}
\hline Leyenda & $\begin{array}{c}\text { Paraan ng } \\
\text { Pananampalataya }\end{array}$ & $\begin{array}{c}\text { Puwersang } \\
\text { Pangkapaligiran }\end{array}$ & Puwersang Panlipunan \\
\hline El Leyenda del Isla Sta. Cruz & Pagdarasal (reza) & & $\begin{array}{l}\text { Paglusob ng mga bandidong } \\
\text { grupo. }\end{array}$ \\
\hline $\begin{array}{l}\text { El Leyenda del Nuestra Señora } \\
\text { de la Virgen del Pilar }\end{array}$ & Pagpruprusisyon (procession) & & Isang relihiyosong pagdiriwang. \\
\hline El Leyenda del Curuan & $\begin{array}{l}\text { Pagbabasa ng Bibliya (liende } \\
\text { biblia) }\end{array}$ & & Pagkarelihiyoso ng mga Moro \\
\hline $\begin{array}{l}\text { Paquichura el Tetuan ya saka su } \\
\text { Nombre }\end{array}$ & Pagbibinyag (bautismo) & & $\begin{array}{l}\text { Ipinagdiwang ang Unang } \\
\text { sentinaryo ng baryo Tetuan. }\end{array}$ \\
\hline El Leyenda del Bunguiao & $\begin{array}{l}\text { Paniniwala sa Panginoon ( } \text { ta cre } \\
\text { con el dios) }\end{array}$ & $\begin{array}{l}\text { Pagkahulog ng bunga ng niyog } \\
\text { sa harap ng pusa. }\end{array}$ & \\
\hline El Leyenda del Sinunuc & $\begin{array}{l}\text { Pagdiriwang ng Pista (celebra } \\
\text { fiesta) }\end{array}$ & & $\begin{array}{l}\text { Pagdiriwang sa araw ng Patron } \\
\text { ng baryo. }\end{array}$ \\
\hline El Leyenda de Sta. Catalina & $\begin{array}{l}\text { Pagtatayo ng Kapilya (hace } \\
\text { capilla/ermita) }\end{array}$ & & Pananampalataya sa Santo. \\
\hline El Leyenda del San Roque & $\begin{array}{l}\text { Pagdarasal (reza) at Pagtatayo } \\
\text { ng Kapilya (hace capilla/ermita) }\end{array}$ & $\begin{array}{l}\text { Paglaganap ng kolera sa baryo } \\
\text { at pagkaligtas ng mga tao laban } \\
\text { sa naturang sakit. }\end{array}$ & \\
\hline El Leyenda del San Jose Gusu & $\begin{array}{l}\text { Pagdarasal (reza) at Pagtatayo } \\
\text { ng Kapilya (hace capilla/ermita) }\end{array}$ & $\begin{array}{l}\text { Pagkamatay ng mga pananim } \\
\text { dahil sa tagtuyot }\end{array}$ & \\
\hline $\begin{array}{l}\text { Paquichura el Mercedes ya Saka } \\
\text { su Nombre }\end{array}$ & $\begin{array}{l}\text { Pagnonobena at pagmimisa } \\
\text { (novena y misa) }\end{array}$ & $\begin{array}{l}\text { Taggutom sa baryo, } \\
\text { pagkakasakit at pagkamatay ng } \\
\text { mga tao dahil sa kolera, at } \\
\text { pagdadakila sa birhen. }\end{array}$ & \\
\hline El Leyenda del Sta. Barbara & Pagbibinyag (bautismo) & & $\begin{array}{l}\text { Ang matalik na kaibigang } \\
\text { Katoliko ng prinsesa na siyang } \\
\text { naging daan sa pagbabago ng } \\
\text { kanyang pananampalataya }\end{array}$ \\
\hline $\begin{array}{l}\text { El Milagro del Sto. na Tabla la } \\
\text { Virgen de la Soledad de } \\
\text { Dolorosa }\end{array}$ & $\begin{array}{l}\text { Pagnonobena at pagrorosaryo } \\
\text { (novena y rosario) }\end{array}$ & $\begin{array}{l}\text { Paglitaw ng imahen ng Santo sa } \\
\text { Tabla. }\end{array}$ & \\
\hline $\begin{array}{l}\text { El Milagro del Patrona del } \\
\text { Bolong Nuestra Señora del } \\
\text { Rosario }\end{array}$ & $\begin{array}{l}\text { Pagdiriwang ng Pista (celebra } \\
\text { fiesta) }\end{array}$ & & $\begin{array}{l}\text { Pagkaligtas ng baryo laban sa } \\
\text { mga sumalakay na Moro }\end{array}$ \\
\hline El Alma Agrecido & $\begin{array}{l}\text { Paniniwala sa muling } \\
\text { pagkabuhay ng kaluluwa } \\
\text { (resureccion del alma) }\end{array}$ & & $\begin{array}{l}\text { Ang aksidente at pagkamatay ni } \\
\text { Graciano. }\end{array}$ \\
\hline El Cirujana & Pangungumpisal (compesa) & $\begin{array}{l}\text { Pagkakaroon ng matinding sakit } \\
\text { ng babae at pagkamatay. }\end{array}$ & \\
\hline
\end{tabular}

Matutunghayan sa Talahanayan 1 ang mga salik na nakaimpluwensiya sa paraan ng pananampalataya ng mga Zamboangueño: (a) puwersang pangkalikasan at (b) puwersang pantao. 


\section{Diskusyon}

\subsection{Puwersang pangkalikasan}

Ang puwersang pangkalikasan ay tumutukoy sa lahat na panlabas na mga puwersa, mga natural na kaganapan o penomena, mga bagay-bagay na hindi kontrolado ng tao o mga tao. Samantalang ang pananampalataya ay isang paniniwala sa isang Diyos. Ito ay siyang kapanatagan sa mga bagay na hinihintay, ang katunayan ng mga bagay na hindi nakikita (Hebreo 11:1). Inaakay ng pananampalataya sa Diyos ang mga tao sa lahat ng kaalaman at sa lahat ng kaganapan at sa buong katapatan sa harapan nila. Narito ang iba't ibang salik pangkalikasan na nakaimpluwensiya sa paraan ng pananampalataya ng mga Zamboangueño batay sa mga leyendang Chabacano ng lungsod Zamboanga.

Pagkahulog ng Bunga ng Niyog sa Pusa - Ang pagkahulog ng bunga ng niyog sa pusa ay isang pangyayari na pinaniniwalaang kagustuhan ng Diyos. Tunghayan ang pangungusap sa ibaba:

Como un acto de Dios, un coco laya ya cay na insima del gato.

(Parang kagustuhan ng Diyos, biglang nahulog sa pusa ang isang bunga ng niyog). (mula sa El Leyenda del Bunguiao).

Inilalarawan mula sa siniping pahayag ang pagkakaroon ng paniniwala sa Panginoon. Sa El leyenda del Bunguiao, ang salik na nakaimpluwensiya sa paraan ng pananampalataya ay isang uri ng puwersang pangkalikasan kung saan ipinalalagay ng matanda sa kuwento na ang pagkahulog ng bunga ng niyog ay kagustuhan ng Panginoon. Naniniwala siya na anomang pangyayaring nagaganap sa kapaligiran ay hindi basta lamang nangyayari kung hindi ito kaloob ng Diyos. Ipinakikita rito ang pagkakaroon ng pananampalataya ng mga Zamboangueño sa Diyos kahit na hindi nila ito nakikita. Ipinalalagay nila na ang bawat kaganapan sa kapaligiran o mga natural na penomena tulad ng pagkahulog ng niyog ay isang akto ng Diyos. Ayon sa Hebreo 11:6, ang pananampalataya ay ang paniniwala sa nag-iisa at tunay na Diyos kahit hindi natin siya nakikita.

Ipinakikita lamang ng mga Zamboangueño na malaki ang kanilang paniniwala sa Panginoon kahit hindi nila ito nakikita. Ipinalalagay nila na may dahilan ang Diyos kung bakit nangayayari ang mga bagay-bagay sa kapaligiran. Ang ganitong uri ng paniniwala ay maaari din nating maihalintulad o maiugnay sa katutubong pananampalataya ng mga ninuno natin kung saan naniniwala din sila kay Bathala bilang pinakamakapangyarihang diyos kahit na hindi naman nila ito nakikita. Nangangahulugan lamang na talagang nakaugat na sa kultura ng mga Pilipino ang pagiging likas na maka-Diyos.

Paglaganap ng Kolera sa Baryo at Pagkaligtas ng mga Tao Laban sa Naturang Sakit - Sa El Leyenda del San Roque ang paglaganap ng kolera sa baryo ang dahilan ng pagdarasal ng mga tao at ang pagkaligtas nila sa sakit na ito ang siyang na nag-udyok sa kanila upang magtayo ng kapilya bilang pasasalamat sa kanilang Santo. Tunghayan ang mga talata sa ibaba:

Antes, el nombre de este barrio amo el Tipung. Un dia el chlolera ya man calayat en este barrio que ya causa el muerte de mucho maga gente.

(Noon ang pangalan ng baryo ay Tipung. Isang araw, lumaganap ang kolera sa baryong ito na siyang naging sanhi ng kamatayan ng maraming tao). (Mula sa unang talata ng El Leyenda del San Roque)

El maga gente ya reza como ya manda canila el padre. Ala tarde, el cholera ya descansa de man kalayat. El maga gente ya cre que el santo amo el ya salva canila des del enfermidad. Para espresa diila gratitude, ya planta el maga gente un ermita para con el santo. 
Mga leyendang Chabacano: Salamin sa pananampalatayang Zamboangueño

(Ang mga tao ay nagdasal dahil na rin sa utos ng pari. Kinahapunan, ang kolera ay nawala. Ang mga tao ay naniwala na ang santo ang siyang nagligtas sa kanila sa kolera. Bilang pasasalamat, nagpatayo ang mga tao ng isang kapilya para sa santo). (Mula sa ikalawa hanggang ikatlong talata ng El Leyenda del San Roque)

Ipinakikita sa mga talata sa itaas ang salik pangkalikasan na siyang nakaimpluwensiya sa pananampalataya ng mga tao sa baryo. Ang pagkakaroon ng sakit na kolera ay isang natural na pangyayari na hindi mapipigilan, ito ang pangyayaring nag-udyok sa mga tao upang magdasal. Mayroon silang matibay na pananampalataya at naniniwalang maililigtas sila ng Panginoon sapagkat noong inutusan sila ng isang pari na magdasal ay hindi sila nag-alinlangan na gawin ito, hindi sila nagtanong, agad nila itong ginawa sapagkat malaki ang tiwala nila na ito ang magliligtas sa kanila laban sa sakit na kumakalat at kumikitil ng buhay. Hindi nga sila nagkamali sapagkat sa ikalawa hanggang ikatlong talata ng leyenda ay nawala ang sakit na kolera kinahapunan. At bilang pasasalamat ay nagtayo sila ng kapilya para sa kanilang santo na siyang pinaniniwalaa nilang nagligtas sa kanila.

Ala tarde, el cholera ya descansa de man kalayat.El maga gente y acre que el santo amo el ya salva canila des del enfermidad.

(Ang mga tao ay nagdasal dahil na rin sa utos ng pari. Kinahapunan, ang kolera ay nawala.Ang mga tao ay naniwala na ang santo ang siyang nagligtas sa kanila sa kolera).

Mapapansin din na isang pari ang nag-utos sa kanila upang magdasal. Ayon kay Julian at Lontoc (2016), naging labis ang paggalang ng mga Pilipino sa mga alagad ng simbahan at sa altar na pinagdarausan ng misa pati na rin sa iba't ibang imahen ng mga santo na naging dahilan ng pagkakaroon ng pista sa bawat lugar ng bansa. Tunay na malaki ang naging epekto ng relihiyong Katolisismo sa buhay ng maraming Zamboangueño. Mula sa pagkakaroon ng katutubong pananampalataya na sumasamba sa kalikasan o sa mga anito at kay Bathala ay natutuhan nilang sumamba sa Diyos ng mga Kristiyano na walang iba kundi si Hesu Kristo.

Pagkamatay ng mga Pananim Dahil sa Tagtuyot - Ipinakikita sa El Leyenda del San Jose Gusu ang salik na nakaimpluwensiya sa pagtatayo ng kapilya ay ang pagkamatay ng mga pananim dahil panahon ng tagtuyot. Tunghayan ang talata sa ibaba:

Tiempo de aquel no hay ulan, el maga siembra ta acaba ya seca y tiene miedo el maga gente si palta el diila comida. El maga gente ya planta un ermita y ya reza para tiene ulan.

(Panahon noon ng tagtuyot, ang mga halaman ay natutuyo kaya natakot ang mga taong magkulang ang pagkain nila. Nagtayo ng kapilya ang mga tao at nagdasal nang ulan). (Mula sa ikalawang talata ng El Leyenda del San Jose Gusu).

Ang tagtuyot o pagtaas ng klima ay isang panahon na sobrang init. Dito maraming mga pananim ang natutuyo sa mga sakahan. Marami ang mga namamatay na halaman dahil sa kakulangan ng tubig. Ito ay isang natural na penomenang nangyayari sa kapaligiran. Inilarawam sa El Leyenda del San Jose Gusu na ang baryo ay nakararanas ng tagtuyot kaya natatakot ang mga tao na magkulang ang kanilang mga pagkain, naisip nila agad na magtayo ng kapilya upang dito ay manalangin ng ulan. Makikita natin sa talatang ito ang pagkakaroon ng matibay na Pananampalataya ng mga Zamboangueño. Sa oras ng matinding pagsubok, ang pananampalatay sa Panginoon ang kanilang tanging sandigan at sandata.

Inilarawan rin sa leyenda na isang araw ay nagkaroon nga ng ulan subalit muli silang sinubok nang tumaas ang tubig, subalit may mag-asawang di nagpatinag. Di sila natakot sa baha at nagtiwala sila sa panginoon kung saan inilarawan sa leyenda na nagpakita sa kanila ang isang santo na si San Jose na siyang nagligtas sa kanila sa kapahamakan.

Un dia ya vene un fuerte ulan en este lugar. El agua ya queda hondo. El maga nativo bien tiene miedo poreso ya sale sila en aquel lugar. Tiene man maridable Viejo y vieja el ya queda na lugar 
cay masquin chiquito lang el diila casa, alto este. Tiene sila fe con el Dios y sabe sila cay este fe amo ay salva canila. Ala madrogada el ulan ya descansa ya y el man maridable ya anda na ermita para reza. Ya mira sila un sombre na distancia. Cuando ya atraca sila con el sombre, ya mira sila un Viejo con largo barbas. El Viejo ya habla: "No tiene miedo cay yo el responsible del de iño salvacion. Yo no hay otro sino si San Jose.

(Isang araw dumating ang malakas na bagyo sa lugar na ito. Ang tubig ay umapaw. Takot na takot ang mga tao kaya umalis sila sa lugar na iyon. Ang mag-asawang matanda ay nagpaiwan dahil kahit na maliit lamang ang kanilang dampa, mataas naman ito. Mayroon silang matinding pananalig sa Diyos at batid nila na ito ang magliligtas sa kanila. Hatinggabi nang tumila ang ulan kaya ang mag-asawa ay pumunta sa kapilya upang magdasal. Nakakita sila ng isang anino sa malayo. Nang sila'y lumapit sa kinaroroonan ng anino, nakita nila ang isang matandang lalaki na may mahabang balbas. Ang matandang lalaki ay nagsalita: "Huwag kayong matakot dahil ako ang magliligtas sa inyo. Ako ay walang iba kundi si San Jose).

Ipinakikita lamang ng mga Zamboangueño kung gaano kalaki ang kanilang tiwala at pananampalataya sa mga santo/patron ng kanilang baryo. kahit gaano pa kalaki ang kanilang problema wala silang pagdadalawang isip na ito ang tutulong sa kanila at ito ang maglalayo sa kanila sa lahat ng kapahamakan. Kaya naman dito sa lungsod Zamboanga napakalaking selebrasyon ang ginagawa ng bawat baryo /barangay kapag sumapit na ang araw ng kapistahan ng kani-kanilang patron na santo bilang pasasalamat.

Ayon sa turo ng Romano Katoliko, ang mga santo ay yaong mga namatay at nakasama ni Kristo sa langit na kinikilala ng simbahan dahil sa pantangi nilang kabanalan at kabutihan. Ang relihiyosong paniniwala na pinagtibay sa konsilyo ng Trent ay nagsasabi na ang mga santo ay dapat hingan ng tulong bilang mga tagapamagitan sa Diyos at ang mga relikya at imahen ng mga santo ay dapat ituring na banal (Mga SantoWatchtower online library https://wol.jw.org/am/wol/d/r27/lp-tg/1101989265).

Pagkagutom sa Baryo Pagkakasakit, Pagkamatay ng mga Tao sa Kolera, at Pagdadakila sa Birhen - Sa leyenda na Paquichura el Mercedes ya Saka su Nombre ipinakikita na nakararanas ang mga tao ng taggutom at maraming tao ang namamatay dahil sa sakit na kolera. Ito ang salik pangkapaligiran na nakaimpluwensiya sa paraan ng pananampalataya ng mga Zamboangueño. Tunghayan ang talata sa ibaba:

\footnotetext{
Desgraciamente, ya queda bien hambre el maga gente na barrio y mucho diila ya acaba muri por causa del enfermedad de cholera. Mucho gayot ya muri por causa del epidemia y ya llega un tiempo, cuando tiene diez gente enterao na un sepultura.
}

(Sa kasamaang-palad, inabot ng taggutom ang baryo, maraming mga tao ang nagkasakit at namatay dahil sa sakit na kolera. Marami ang nasawi dahil sa epidemya at may pagkakataon pang inilibing sa iisang puntod ang sampung katao). (Mula sa ikatlong talata ng Paquichura el Mercedes ya Saka su Nombre).

Inilalarawan sa leyenda na dumanas ng matinding taggutom ang mga tao, at bukod diyan marami din sa kanila ang namatay dahil sa epidemya. Ang kolera ay isang nakahahawa at nakamamatay na sakit. Mayroong iba't ibang uri ng kolera, ngunit lahat ng ito ay nakahahawa. Ang sanhi ng sakit na ito ay ang bacillus bacteria na nabubuhay sa maruruming tubig. Dahil sa matinding pagsubok na ito pumunta ang mga tao sa simbahan upang magnobena ng siyam na araw.

Mula sa Espanyol na novena o pagsisiyam, ang nobena ay isang panatang Kristiyano na kailangang gawin nang paulit-ulit sa loob ng siyam na araw. Malimit na isinasagawa ito sa paraan ng pagrorosaryo, pag-eestasyon, o pagsisimba nang siyam na araw. Ang nobena ay isang sakripisyo para sa katuparan ng isang kahilingan o isang paraan ng pasasalamat sa tinamong magandang kapalaran.

Ipinakikita lamang sa El Leyenda del Mercedes na ang mga tao ay nagnobena bilang paraan nila sa pagsugpo

78 Consortia Academia Publishing (A partner of Network of Professional Researchers and Educators) 
ng naturang sakit. Ramdam nila na ang tanging makapagliligtas sa kanila ay walang iba kundi ang kanilang pananampalataya. Sa mga matitinding pagsubok na kinahaharap sa buhay, ipinakikita lamang dito na ang tanging takbuhan ng mga Zamboangueño laban sa mga pagsubok ay ang paniniwala sa Panginoon. Isinasaad sa leyenda na kinahapunan ay nawala na ang sakit na kolera. Bilang pasasalamat ay nag-alay sila ng misa sa birhen ng Mercedes.

Con este calamidad, el maga gente ya resolva con mas rezo. Ya anda sila na iglesia para reza. Ya hace sila novena por nueve dias y despues de nueve dias, el epidemia ya disaparece. Na pensamiento del maga gente, este el resulta del diila promesas, poreso ya oferece sila misa de gracias en honor de la Virgen de la Mercedes.

(Dahil sa naturang kalamidad, nagdasal ang mga tao. Pumunta sila sa simbahan upang manalangin. Nagnobena sila ng siyam na araw at matapos ang ikasiyam na araw ng pagnonobena ay nawala ang epidemya. Ipinalagay ng mga tao na ito ay bunga ng kanilang panata, kaya nag-alay sila ng isang misang pagpapasalamat bilang parangal sa mahal na Birhen ng Mercedes).

Para sa mga Katoliko, ang banal na misa ay ang pinakamahalaga at pinakamataas na uri ng panalangin. Ayon sa doktrina ng simbahang Katoliko, ang pagdalo sa misa at pakikibahagi sa komunyon ay isang obligasyon upang mabayaran ang mortal na kasalanan, at kailangang gawin linggo-linggo o sa mga banal na araw ng obligasyon. Ito ang ginawa ng mga tao sa barangay Mercedes bilang pagdadakila sa mahal na birhen ng Mercedes dahil naniniwala ang mga tao na ito ang nagligtas sa kanila sa matinding pagsubok na kanilang naranasan.

Ayon kina Julian at Lontoc (2016), ang mga Pilipino noon at tinuruan ng mga misyonerong Espanyol na magdasal, at magsimba, at magbasa ng Bibliya, banal na aklat ng mga katoliko. Natutuhan din nila ang pagdarasal ng orasyon nang sama-sama tuwing ikaanim ng hapon, pagnonobena, pagrorosaryo, at pagsama sa mga prusisyon na siyang lalong nagpatibay sa pagbubuklod ng pamilya. Ipinakilala rin nila ang mga imahen ng mga santo.

Paglitaw ng Imahen ng Santo sa Tabla - Ipinakikita sa leyenda ng El Milagro Del Santo Na Tabla La Virgen De La Soledad De Dolorosa ang paglitaw ng imahen ng Santo Tabla na siyang salik na nakaimpluwensiya sa pagnonobena at pagrorosaryo ng mga Zamboangueño. Tunghayan ang talata sa ibaba:

Cuando ta baña ya el vieja, ya puede ele mira tiene figura de Santo ta aparece na tabla. Cuando ya mira el vieja con el Santo ya ulbida ya le el bañada. Ya volve ele y ya llama con el maga vecinos. El maga gente ya hace ofricimiento como novena y Sto. Rosario. Ya continua el maga gente, y el vieja tambien con el diila rezada.

(Nang naliligo na ang matandang babae, naaaninaw niya ang larawan ng santong lumilitaw sa tabla. Nang makita ng matanda ang santo, nakalimutan niya ang paliligo. Umuwi siya at tinawag ang kanyang mga kapitbahay. Ang mga tao ay gumawa ng pag-aalay gaya ng pagnonobena at pagrorosaryo. Ipinagpatuloy ng mga tao pati na rin ng matandang babae ang kanilang pagdarasal). (Mula sa ikalawang talata ng El Milagro Del Santo Na Tabla La Virgen De La Soledad De Dolorosa).

Inilarawan sa leyendang ito na nagpakita sa matandang babae ang Birhen. Ang Birhen ay may iba't ibang katawagan depende sa lugar kung saan siya nagpakita o nagkaroon ng aparisyon subalit tumutukoy lamang ito sa nag-iisang si Birheng Maria. Napakalaki ng paggalang ng mga Katoliko kay Birheng Maria. Kinikilala nila ito bilang "Ina ng Panginoong Hesus". Kaya nang makita ng matandang babae ang pigura ng Mahal na Birhen ay agad siyang umuwi at ibinalita ito sa kanyang mga kapitbahay. Ipinakikita rin sa leyendang ito na agad naniwala ang mga tao sa aparisyon ng Mahal na Birheng Maria, wala silang pagdadalawang-isip sa katotohanan ng pangyayaring ito. Agad silang nag-alay ng nobena at nagrosaryo. Makikita natin kung gaano kalaki at katindi 
Reyes, A.

ang debosyon ng mga Zamboangueño sa Mahal na Birhen, talagang dinadakila nila ito.

Maaaring natutuhan ng karamihan sa mga Pilipinong Katoliko ang pananampalataya mula sa kanilang debosyon sa Birhen Maria. Ito ay nakasaad na sa kasulatan kung saan sinasabi na si Maria ang dakilang halimbawa ng pananampalataya sa pamamagitan ng kanyang pagpayag sa "announciation" si Maria ay naging modelo ng pananampalataya (AMB 35; cf CCC 148). Kung titingnan natin ang mga mesahe ng mahal na birhen sa kanyang mga aparisyon, mapapansin nating palagi niyang kinokonekta ang mga prediksyon ng mga paparating na kalamidad - "kung ang mga tao ay hindi magdasal" (Fuellenbach, 1992).

Pagkakaroon ng Matinding Sakit ng Babae at Pagkamatay - Sa leyenda na El Cirujana ang pagkakaroon ng matinding sakit ng babae ay siyang dahilang ng kanyang pangungumpisal. Tunghayan ang siniping talata:

El cirujana antes joven pa y uno lang el anak cuando ele ya enferma. Ya queda gayot ele malismo y ya dale ya con ele compesa el padre. Despues ya muri ya le.

(Ang seruhana noon ay bata pa at iisa pa lang ang anak nang siya'y nagkasakit. Naging malubha siya at pinakumpisal na siya ng pari. Pagkatapos siya ay namataya). (mula sa ikatlong talata ng El Cirujana).

Inilalarawan sa leyendang ito na ang babae ay nagkaroon ng malubhang sakit kaya ipinatawag ang pari upang siya ay makakumpisal bago siya malagutan ng hininga. Naniniwala ang mga katolikong Zamboangueño na kapag ang isang tao ay malapit nang mamatay ay kailangan niyang humingi ng kapatawaran sa Panginoon habang may nalalabi pa siyang oras sapagkat kapag ang isang tao ay patay na wala na siyang kakayahan at karapatang humingi ng tawad sa kanyang mga kasalanan. Hindi na siya pakikinggan ng Panginoon. Ang pangungumpisal na inilarawan sa leyenda ay isang bahagi sa tinatawag na anointing of the sick. Ang matandang paniniwala ng mga Pilipino noon ay napalitan ng bagong paniniwalang itinuro ng mga misyonerong Espanyol. Nanguna sa pagpapalaganap ng paniniwalang ito ang mga misyunerong Agustino na kasama ni Legazpi sa kanyang pagdating sa Pilipinas noong 1565 sa pangunguna ni padre Andres de Urdaneta. Sinundan pa ito ng mga Pransiskano noong 1577, ng mga Heswita noong 1581, ng mga Dominikano noong 1587, at mga Recoletos noong 1606. Nagpatayo din ang mga misyonero ng mga simbahan. Dito itinuro ang kahalagahan ng mga sakramento tulad ng binyag, kasal, pagdalo sa misa, kumpil, pangungumpisal, at pagpapabendisyon sa mga maysakit at namatay (Julian at Lontoc, 2016).

\subsection{Puwersang pantao}

Ito ay nagbibigay diin sa puwersang sumasaklaw sa lahat ng kapaligirang panlipunan, pang-institusyon, pangkultura, mga penomenang panlipunan na tao mismo ang may gawa at kung paano sila nakaaapekto at naaapektuhan, samantalang ang pananampalataya ay tumutukoy sa "paniniwala sa, debosyon sa, o tiwala sa isang tao o isang bagay, na walang patunay sa lohika". Tumutukoy din ito sa pananampalataya bilang "paniniwala o debosyon sa Diyos". Tunghayan ang iba't ibang salik pantao na nakaimpluwensiya sa paraan ng pananampalataya ng mga Zamboangueño batay sa mga leyendang Chabacano ng lungsod Zamboanga.

Paglusob ng mga Bandidong Grupo - Sa El leyenda de Sta. Cruz ang salik na nakaimpluwensiya sa pananampalataya ng babae ay ang paglusob ng mga bandidong grupo sa isang barko. Tunghayan ang talata sa ibaba.

Cuando ya pundia el barco na isla, el maga tripulante y pasajero ya abaja para busca cosa puede sila come. Un grupo de bandido ya ataca canila. Con el capitan, con el negociante y otro maga pasajero ya mata estos maga bandidos

(Nang dumaong ang sasakyang-dagat sa pulo, ang mga tripulante at pasahero ay nanaog para maghanap ng pagkain. Isang pangkat ng mga bandido ang sumalakay sa kanila. Ang kapitan, ang negosyante, at ang iba pang mga pasahero ay pinagpapatay ng mga bandido). (Mula sa 
ikalawang talata ng El Leyenda del Sta. Cruz).

Ang siniping payahag sa itaas ay naglalahad ng pagkakaroon ng puwersang pantao kung saan ang mga bandidong grupo ay gumawa ng kasamaan laban sa mga pasahero ng barko. Ang pagpatay ng mga tulisan sa mga pasahero ng barko at ang pagtatangkang pangingidnap sa babaeng pasahero upang gawing asawa at alipin ng kanilang pinuno. Ang mga pangyayaring ito ang siyang naglagay sa babae sa matinding panganib na siya namang naging dahilan ng pagpapakita niya ng pananampalataya sa pamamagitan ng pagdarasal sa Panginoon. Sa pangyayaring ito sa buhay ng babae, naniniwala siya na wala nang ibang makapagliligtas pa sa kanya kundi ang pananampalataya niya sa Panginoon.

Ang paglusob ng mga rebelde sa mga barko ay talagang laganap noon sa lungsod ng Zamboanga. Maraming mga inosenteng tao ang tinatangay ng mga bandido o rebelde upang ipatubos sa kanilang mga pamilya, sa ganitong mga sitwasyon ay nagkakaisa talaga ang mga Zamboagueño sa pagdarasal sa Poong Maykapal at ilang beses na ring napatunayan na walang imposible sa kapangyarihan ng panalangin at pagtitiwala sa Panginoon. nakasanayan na ng mga tao sa Zamboanga na gawing sandata ang pagdarasal laban sa iba't ibang pagsubok sa buhay.

Isang Relihiyosong Pagdiriwang - Maraming mga relihiyosong pagdiriwang ang bahagi ng pananampalatayang Kristiyano. Ang lungsod Zamboanga ay isa sa mga lugar sa Pilipinas na matapat na gumagawa at nag-oobserba sa mga pagdiriwang panrelihiyon na ito. Isa na rito ang Semana Santa. May iilan ding mga mahalagang kaganapan ng pagdiriwang ang makikita tuwing "Semana Santa", isang ekspresyong Zamboangueño tungkol sa Seman Santa ang panonood ng pelikula tungkol sa Panginoon at ang kanyang mga turo, Visitaciones de las Inglesias, prusisyon, nobena at ang pag-akyat at pagdarasal sa Estaciones de Cruz ng Mt. Pulong Bato. Ang prusesyon ng lungsod na kilala bilang Santo Entierro (Holy Funeral) sa Biyernes Santo ay nagha-highlight sa pagdiriwang ng panahon ng Kuwaresma. Ipinagdiriwang sa mga barangay Sta. Maria, Tetuan, at ang Immaculate Conception Cathedral Parish, ang Santo Entierro ay isang prusisyon ng mga imahen ni Kristo, marami sa mga istasyon ng krus, kasama ang mga pangunahing lansangan ng lungsod, na naglalarawan ng kanyang mga huling oras bago, sa panahon, at pagkatapos ng Paglilibing. Ang pagdiriwang na ito ay dinadaluhan ng libo-libong mga Zamboangueño kung saan ang daanan patungo sa itaas ng bundok kung saan naroon ang krus mayor ay punong-puno ng mga tao. Napakataas at napakatarik ng daanan, maaari kang mahulog sa bangin at mapahamak subalit hindi mo makikita ang pag-aalinlangan ng mga tao, patuloy sila sa pagdarasal hanggang maabot nila ang pinakatuktok ng Krus Mayor. Isang katuparan ang kanilang nararamdaman sa tuwing nararating na nila ang tuktok ng bundok, dito nagsisindi sila ng kandila sa krus mayor at taimtim na humihingi ng kapatawaran sa lahat ng kasalanang nagawa. Isang matinding sakripisyo ang kanilang ginagwa at kapansin-pansin na bawat taon ay lalong dumadami ang mga mananampalataya na umaakyat at nag-eestasyon sa krus bilang panata nila sa Panginoon.

Ipinakikita sa El Leyenda del Nuestra Señora de la Virgen del Pilar na nagkaroon ng prusisyon dahil sa isang relihiyosong pagdiriwang. Ito ang salik na nakaimpluwensiya sa paraan ng pananampalatayang inilarawan sa leyendang nabanggit. Tunghayan ang talata sa ibaba:

\section{El historia ya principia cuando el imagen del Nuestra Señora de la Virgen del Pilar ya marca na dindin del moralla. Tiene aquel un relihioso ocacion poreso ya tene tambien procession para del Nuestra Señora de la Virgen del Pilar.}

(Ang kuwento ay nagsimula nang ang imahen ng Nuestra Señora de la Virgen del Pilar ay naukit sa pader ng moog. May isang relihiyosong pagdiriwang noon kaya nagkaroon din ng prusisyon para sa Nuestra Señora de la Virgen del Pilar). (Mula sa unang talata ng el Leyenda del Nuestra Señora de la Virgen del Pilar).

Inilalahad sa leyendang ito na nagkaroon ng prusisyon dahil may isang relihiyosong pagdiriwang. Ang pagdiriwang na tinutukoy sa leyenda ay ang pista ng Mahal na Birhen ng Pilar. Tuwing sumasapit ang ika-12 ng 
Reyes, A.

Oktubre ay ipinagdiriwang ng mga Zamboangueño ang kapistahan ng Mahal na Birhen ng Pilar. Nagkakaroon ng parada, mga sayawan at ang pagkakaroon ng prusisyon at misa ay ang pinakapangunahing haylayt ng pagdiriwang. Ito ay bilang pasasalamat ng mga Zamboangueño Kristiyano man o Muslim, sa birhen ng Pilar sa lahat ng kanilang mga kahilingang natanggap.

Ayon kina Yap-Aizon et al. (2016), ipinakikita ng mga Zamboangueño ang kanilang matinding debosyon sa imahen ng Fort Pilar. Kung mayroong mga panganib mula sa sangkatauhan o kalamidad man, ang kanilang kaligtasan ay palaging dahil sa Birhen del Pilar. Ito ay naging debosyon ng mga Espanyol at Katutubo kaya idineklara ng mga Espanyol ang Oktubre 12 bilang holiday o walang pasok. Ang kamangha-manghang prusisyon na nagsisimula sa simbahan patungong Fort ay isinasagawa na tuwing sumapit ang araw na iyon. Kapag nakarating na ang prusisyon sa Fort "salve" o salutasyon ang inaawit bilang pasasalamat sa mga biyayang natanggap mula sa Birheng Maria. Nakatala na ang imahen ng Birhen del Pilar ng lungsod Zamboanga ay orihinal na inilagay sa tsapel sa loob ng dingding ng Fort. Batay sa ginawang estimasyon, ang imahen ay maaaring inilipat sa pintuan noong 1734. Naniniwala ang mga tao noon na ang imahen ay inilagay ni Melchor de Vera bilang bahagi ng ginagawang konstruksyon. Noon lamang 1719 nang nagkaroon ng muling konstruksyon na ang iginagalang na birhen sa Fort ay inilagay bilang religious centrepiece sa pasukan nito. Ang fort ay pinaniniwalaang nagmimilagro mula kay Birhen del Pilar na inilagay noong 1734 bilang religious centrepiece sa itaas ng silangang bahagi ng pader. Bilang resulta ng iba't ibang milagro ng Birhen del Pilar, ang imahen ay ginawang bukas na dambana na may altar. Ang mga milagro ng dambana ay hindi lamang pinaniniwalaan ng mga Kristiyano kung hindi pati na rin ng iilang mga Muslim na nakaramdam ng naturang mga pangyayari. Pumupunta ang iilang mga Muslim na natibo na ng lungsod sapagkat naniniwala sila na ang lugar na ito ay dating dasalan ng mga Muslim bago pa dumating ang mga Kastila. Karamihan din sa mga Muslim na pumupunta dito ay dahil mismong ang mga magulang nila ang nagpakilala sa kanila sa ganitong paniniwala. Nagdarasal at humihiling sila kay Maria sa tulong ni Allah at karamihan sa kanila ay natupad ang kanilang mga kahilingan. Ang dambana ay tinaguriang multi-religious. Ito ay maaaring di pangkaraniwan subalit hindi kailanman hiwalay o kakaibang penomena. Ang tagapagtanggol ng lungsod, ito ang Birhen del Pilar para sa mga Zamboangueño.

Pagkarelihiyoso ng mga Moro - Matatagpuan sa Koran ng mga Muslim ang kanilang mga aral. Naglalaman ito ng mga kautusang panrelihiyong dapat sundin ng mga Muslim. Malaki ang naging impluwensiya ng pananampalatayang Islam sa buhay ng mga Pilipinong Muslim. Kabilang sa mga ito ang paggamit ng wikang Arabic at ang hindi pagkain ng karne at dugo ng baboy dahil sa paniniwalang ito ay marumi. Mayroon silang pistang panrelihiyon katulad ng Hariraya Puasa, Araw ng Pasasalamat at hudyat ng pagsisimula at pagwawakas ng buwan ng Ramadan; Nipso, katumbas ng Araw ng mga Patay ng mga Kristiyano; Maulud, ang Araw ng Pagsilang ni Mohammed (Julian at Lontoc, 2016).

Sa El Leyenda del Curuan ang pagiging relihiyoso ng isang Moro ang siyang salik kung bakit siya nagbabasa ng Koran na inilarawan sa leyendang binaggit. Tunghayan ang pangungusap sa ibaba:

Bien religioso gayot estos maga Moro y ta pasa sila diila tiempo liendi biblia que ta llama "Koran"

(Napakarelihiyoso talaga nitong mga Moro at pinalilipas nila ang kanilang panahon sa pagbabasa ng bibliya na tinatawag na Koran mula sa unang talata ng El Leyenda del Curuan).

Ipinakikita sa leyenda ang katangian ng mga Moro bilang napakarelihiyoso. Ang pagiging relihiyoso ay maiuugnay naman sa mga gawaing espiritwal ng isang tao dulot ng pagkakaroon niya ng pananampalataya. Isinasaad sa siniping pangungusap sa itaas ang pagbabasa ng isang Moro ng Koran tuwing wala siyang ginagawa. Nangangahulugan lamang na matindi ang debosyon sa relihiyong Islam ng mga Muslim ng Zamboanga. Lalo nilang hinahasa at pinapamilyar ang mga nakasaad sa banal na aklat o Koran na siyang patnubay nila sa paggawa ng kabutihan sa buhay. Ayon sa katuruan ng Islam ang bawat letra ng Koran ay may katapat na gatimpala. 
Mga leyendang Chabacano: Salamin sa pananampalatayang Zamboangueño

Ipinagdiwang ang Unang Sentinaryo ng Baryo Tetuan - Sa Paquichura el Tetuan ya Saka su Nombre, ang salik na nakaimpluwensiya sa pagbibinyag ay ang pagdiriwang ng unang sentinaryo ng baryo. Tunghayan ang talata sa ibaba:

El primer padre parroco era el padre Jesuita Ramon Barva y el primer bautismo bajo del de suyo administracion ya socede el fecha 3 de Enero, del año 1963, que ya senala el fundacion official del barrio de Tetuan. El primer sentenario del barrio de Tetuan ya celebra tamen por fin el dia de Enero del año 1963.

(Ang unang kura paroko ay isang paring Hesuwita na si padre Ramon Barva at ang kauna-unahang pagbibinyag sa ilalim ng kanyang pamamahala ay ginanap noong Enero 3, 1863 na siyang pasimula ng pagtatag $n g$ baryo ng Tetuan. Ang unang sentinaryo ng baryo Tetuan ay ipinagdiwang noong Enero 3, 1963). (Mula sa huling talata ng Paquichura el Tetuan y saka su Nombre).

Ipinakikita sa leyendang ito na bilang panimula sa pagtatag ng baryo ng Tetuan ay nagkaroon din ng pinakaunang pagbibinyag o bautismo sa ilalim ng administrasyon ng bagong kura paroko. Ang ganitong gawain ng simbahan ay talagang ginagawa hanggang sa kasalukuyang panahon. Tulad nga sa nabanggit sa naunang tsapter ay nagkakaroon talaga ng mass baptism ang bawat parokya ng barangay kapag sumasapit ang kanilang kapistahan. Libre itong isinasagawa bilang pasasalamat sa patron at upang maraming mga bata ang makatanggap ng sakramento ng binyag at siya ay magiging kaisa ng Diyos. Naniniwala ang mga Zamboangueño sa kahalagahan ng sakramento ng binyag upang magkaroon ng patnubay ang isang tao sa kanyang buhay mula sa Panginoon. kung hindi kasi nabinyagan ang isang Kristiyano naniniwala din ang mga nakatatanda na kapag siya ay namatay, hindi siya makaaakyat sa langit sapagkat hindi siya nakalista sa Panginoon, wala siyang pangalan dahil hindi siya nabinyagan. kaya nga ang mga sanggol na ipinapanganak na patay ay binibinyagan pa rin bago ilibing.

Ang bautismo ay isang sakramento o ritwal ng mga Kristiyano na ginagawaran ng paglulubog sa tubig o pagbubuhos ng banal na tubig sa may ulunan ng sanggol o maging nasa-edad na, bilang tanda ng pagiging bahagi niya sa pamayanang Kristiyano. Sa pamamagitan nito, inaasahang magiging mabuting Kristiyano ang binibinyagan. Tinatawag itong binyag sa Simbahang Katoliko Romano, subalit ginagamit ang salitang buhos kapag hindi pari ang nagsasagawa ng pagbibinyag. Isang pagdadalisay na pampananampalataya ang pagbabautismo sa pamamagitan ng banal na tubig. Ginagawa ang seremonyas na ito ilan pang mga relihiyon, bukod sa Katolisismo at Kristiyanismo, katulad ng Mandaenismo, Sikhismo at ilang mga sekta ng Hudaismo. Bukod sa paglulubog, isa ring paghuhugas sa pamamagitan ng tubig ang pagbabautismo na sumasagisag sa pagkakatanggal ng mga kasalanan ng isang tao, at sa pagsapi ng taong ito sa mag-anak ng Diyos, at sa kaniyang pagiging kaisa sa pagkamatay ni Hesus (dahil sa kasalanan ng tao) at sa muling pagkabuhay ni Hesus (sumasagisag sa bagong buhay) (Pagbibinyag, binyagan https://www.lds.org/scriptures/gs/baptism-baptize?lang=tgl).

Pagdiriwang sa Araw ng Patron ng Baryo - Inilarawan sa El Leyenda del Sinunuc ang pagdiriwang sa araw ng Patron bilang salik na nakaimpluwensiya sa paraan ng pananampalataya na pagdiriwang ng Pista ng mga Zamboangueño. Tunghayan ang talata sa ibaba:

Un aga, fecha 28 de Mayo, 1900 mientras el maga Cristiano ta celebra diila fiesta, un hombre ya grita "Ta quema el maga Moro con el ermita y maga casa"!

(Isang Umaga, ika-28 ng Mayo, 1900 habang ang mga Kristiyano'y nagdiriwang ng kanilang pista, isang lalaki ang sumugaw "Sinusunog ng mga Moro ang kapilya at ang mga bahay")! (Mula sa ikaapat na talata ng El Leyenda del Sinunuc).

Isinasaad sa talata sa itaas na nagdiriwang ng pista ang mga Kristiyano. Ang pagdiriwang ng pista ay isang 
selebrasyon bilang pasasalamat sa patron ng naturang lugar. Ipinakikita rin sa leyenda na ang mga Kristiyano at Muslim sa baryong iyon ay hindi nagkakasundo kaya wala silang kapayapaan. Ang pagkakaroon ng pista ay nagpapakita na ang mga Kristiyanong Zamboangueño ay nakasandig at nagtitiwala sa gabay at tulong ng kanilang patron. Sa mga kaguluhang nangyayari sa baryong iyon ang kanilang naging takbuhan at pinaniniwalaang magproprotekta sa kanila ay ang kanilang patron kung saan humihiling din sila na magkaroon na rin ng kapayapaan sa naturang baryo, kaya bilang pasasalamat at pagpapakita ng kanilang pananampalataya ay nagdaraos sila ng pista para dito. Kaya nga ang patron ng barangay Sinunuc ay Our Lady of Peace and Good Voyage at ipinadiriwang ng mga taga-Sinunuc ang kapistahan ng kanilang patron tuwing Mayo 28.

Malaki ang impluwensya ng Kristiyanismo lalong-lalo na ang simbahang Katoliko Romano sa kasulukuyang kultura ng Pilipinas. Makikita ito sa ating mga pang araw-araw na kilos at gawi. Maging ang ating mga paniniwala ay lubos na naapektuhan ng simbahan. Ang pagdaraos ng mga kapistahan, ay isang tradisyon na may malalim na impluwensyang Kristiyano. Nang dumating ang mga Kastila dala ang relihiyong Kristiyanismo, malugod natin itong tinanggap. Kaalinsabay ng paglaganap ng Kristiyanismo ay ang pagtatalaga ng isang santong patron para sa isang bayan o pamayanan. Para sa mga patron ay nagtalaga tayo ng isang araw ng pasasalamat at ito ay kilala bilang araw ng pista (https://noypi.blogspot.com/impluwensya-ng-kristiyanismo).

Pananampalataya sa Santo - Ang pananampalataya ng ating mga sinaunang ninuno noon ay maitututuring na Paganismo o Animismo, naniniwala sila na ang lahat ng bagay ay may kaluluwa o espiritu. Kasama sa mga bagay na may kaluluwa ang mga hayop, halaman, bato, mga bundok, mga ilog, at mga bituin. Naniniwala ang mga animista na ang bawat anima ay isang makapangyarihang espiritu na maaaring tumulong o manakit sa tao at dapat silang sambahin, katakutan at kilalanin sa iba't ibang kaparaanan. Ang Animismo ay isang katutubong relihiyon na nasa mundo na sa loob ng libo - libong taon, at ang mga naniniwala sa relihiyong ito ay itinuturing na diyos ang mga hayop, bituin, at lahat ng uri ng idolo at nagsasanay ng espiritismo, pangkukulam, panghuhula at astrolohiya.

Nang dumating ang mga Kastila ang matandang paniniwala ng mga Pilipino noon ay napalitan ng bagong paniniwalang itinuro ng mga misyonerong Espanyol. Isa na rito ang pagdarasal ng orasyon nang sama-sama tuwing ikaanim ng hapon, pagnonobena, pagrorosaryo, at pagsama sa mga prusisyon lalong nagpatibay sa pagbubuklod ng pamilya. Ipinakilala rin nila ang mga imahen ng mga santo. Kaya naman madaling natanggap at isinabuhay ng mga Pilipino ang bagong paniniwala na ipinakilala sa kanila ng mga mananakop na Espanyol dahil halos walang pinagkaiba ang pagsamba at paniniwala nila sa mga anito at ang pagsamba at paniniwala nila sa mga santo. Kaya naman ang paniniwala sa mga santo/santa ay malaon ng nakaugat sa kultura ng mga Pilipino at hanggang sa kasalukuyang panahon ay matibay pa rin ang pananampalataya ng mga Zamboangueñong Katoliko sa kanila.

Sa El Leyenda del Sta. Catalina, ipinakikita na ang salik na nakaimpluwensiya sa paraan ng pananampalataya ng mga Zamboangueño ay ang pananampalataya o pananalig ng mga tao sa santo ng baryo. Tunghayan ang talata sa ibaba:

Tiene tamen un mujer ya queda alli, el nombre si Catalina. Ya cumbida le con el maga gente para tiene un miting para hace sila un capilla. Poreso, ya nombra sila el lugar Sta. Catalina porcausa de santo ya icha alli. Hasta ahora, Sta Catalina el nombre del lugar.

(Mayroon namang isang babaeng nakatira doon na ang pangalan ay Catalina. Inimbitahan niya ang mga tao na dumalo sa isang pulong upang makapagpagawa sila ng isang kapilya. Bumili sila ng isang santo at ang pangalan ng santo ay Sta Catalina. Kaya, pinangalanan nila ang lugar na Sta. Catalina dahil sa santong inilagay roon. Hanggang ngayon, Sta. Catalina pa rin ang pangalan ng lugar). (Mula sa huling talata ng El Leyenda del Sta. Catalina)

Isinasaad sa leyenda na isa sa mga babaeng nakatira sa baryo ang nagpatawag ng pagpupulong upang pag-usapan ang pagpapatayo nila ng kapilya. Ang kapilya ay kilala rin sa tawag na simbahan ng bawat baryo. Ito 
ang tinaguriang tahanan ng Panginoon, dito pumupunta ang mga tao para magdasal. Para sa karamihan ng mga Pilipino ang simbahan ay sadyang pamilyar na, para sa iba ang simbahan ay nangangahulugang gusali kung saan pumupunta ang mga tao para magdasal. May iba naman, kapag sinabing simbahan ay agad naiisip ang imahen ng obispo, pari, mga mananampalataya o mga organisasyon sa simbahan. Dagdag pa, noong sinaunang panahon kapag sinabing simbahan ang tinutukoy agad ay ang simbahan ng Katoliko, subalit sa panahon ngayon, ito ay maaaring tumukoy na sa iba't ibang simbahan ng iba't ibang relihiyon (CBCP, 1997).

Sa pagsasalaysay ng leyenda sa itaas, hindi tumutol ang mga tao sa pagpapatayo ng kapilya, isinasaad dito na bumili pa sila ng imahen ng santo upang ilagay sa loob ng kapilya. Ang ganitong uri ng akto ay nagpapakita lamang na ang mga Zamboangueño ay may pananalig at pananampalataya din sa santo. Para sa mga Katolikong Zamboangueño, ang mga santo ay bahagi ng kanilang pananampalataya. Naniniwala sila na ito ang tumutulong sa mga tao sa pamamagitan ng pagsisilbi nitong tulay tungo sa Panginoon. naniniwala ang mga Zamboangueño na dahil malapit ang mga santo sa ating Panginoon ay kaagad nitong pinakikinggan ang kanilang mga dasal. Kaya naman maraming mga mananampalatayang Katoliko ang nagdarasal din sa mga santo at humihiling ng devine intervension upang sila ay tulungan sa kanilang mga hiling patungo sa Panginoon.

Ang Matalik na Kaibigang Katoliko - Ipinakikita sa El Leyenda del Sta. Barbara ang salik na nakaimpluwensiya sa pagpapabinyag ni Barbara ay ang matalik na kaibigang katoliko na siyang naging daan sa pagbabago ng kanyang pananampalataya.

El princesa Barbara tiene un Catolico amigo. Ele Bueno y bien fiel. El princesa ya aprende ya ama con este mujer como su Hermana. Ta anda sila na un lugar donde el rey jende puede mira canila. Este mujer ta habla todo por causa del di suyo religion. Ta dale le con el princesa maga libro Catolico que ta le el princesa durante el di suyo tiempo no hay le que hace.

(Si prinsesa Barbara ay may matalik na kaibigang Katoliko. Siya ay mabait at tapat na kaibigan ni Barbara. Natutunan na siyang mahalin ni Barbara na parang tunay na kapatid. Pumupunta sila sa isang tagong lugar kung saan hindi sila natutunton ng hari. Sinabi ng kaibigan sa prinsesa ang lahat-lahat tungkol sa kanyang relihiyon. Binibigyan niya ng mga Katolikong aklat si Barbara na binabasa naman ng prinsesa sa mga oras na wala siyang ginagawa). (Mula sa ikatlong talata ng El Leyenda del Sta. Barbara).

Isinasaad sa leyenda na minahal ni Barbara ang kanyang kaibigan na parang tunay na niyang kapatid. Ito ang instrumento sa pagbabago ng kanyang loob ukol sa kanyang pananampalataya. Masasabi na ang matalik na kaibigang ito ay may matinding pananampalataya sa relihiyong katoliko sapagkat isinasaad sa leyenda na dinadala niya si Barbara sa simbahan, binibigyan din niya ito ng mga aklat ng Katoliko, at sinasabi din niya dito ang lahat-lahat tungkol sa relihiyong Katoliko. Batay sa paglalarawan, ito ay mga katangian ng isang relihiyosong tao o may malakas na pananampalataya sa Panginoon. Dahil sa matalik na kaibigan niyang ito ay nagbago ang loob ni Barbara sa kanyang dating pananampalataya at ito ay nagpabinyag sa relihiyong Katoliko.

Ang mga tauhan sa leyenda ay mga maaaring mga representasyon lamang sa kung gaano katatag ang pananampalataya ng isang Zamboangueño. Para sa relihiyong Islam ang pagpapabinyag sa ibang relihiyon ay nangangahulugang pagtakwil na rin kay Allah, isa itong napakalaki at mabigat na kasalan kung saan humahantong ito sa pagtatakwil ng magulang o ng pamilya sa kanilang anak o ng sinomang miyembro ng pamilya na nagpabinyag ng ibang relihiyon. Ang ganitong pangyayari ay isang kahihiyan para sa mga magulang dahil ito ay maaaring mangahulugang di nila napalaki nang maayos o di nila nagabayan ang kanilang anak. Kaya naman makikita na dahil sa ginawa ni Barbara ay di napigilan ng kanyang ama ang galit nito kaya humantong ito sa kamatayan ni Barbara sa mismong kamay ng kanyang amang hari.

Si Barbara ya habla con su tata cay ya bautisa ya con ele na iglesia Catolico, cuando ya oi ese el rey, ya saca le su espada y ya curta le el pescueso di Barbara por causa de su rabia 
(Sinabi ni Barbara sa hari na bininyagan na siya sa simbahan ng Katoliko. Sa sobrang galit ng hari nang marinig ito, kinuha niya ang kanyang espada at pinutol ang leeg ni Barbara).

Pagkaligtas ng Baryo Laban sa mga Sumalakay na Moro - Ipinakikita sa leyenda ng El Milagro del Patrona del Bolong Nuestra Señora del Rosario na ang pagkakaligtas ng baryo laban sa mga sumalakay na Moro ay siyang salik na nakaimpluwensiya pagdiriwang ng pista na isa sa paraan ng pananampalataya ng mga Zamboangueño. Tunghayan ang mga talata sa ibaba:

Un noche bien iscuro, mucho vinta de moro na canto del aplaya. El diila intencion derrepente ataca con el maga Cristiano.

(Isang gabi, maraming Bangka ng mga moro ang papalapit sa baybayin ng Bolong. Nagtatangka silang sumalakay sa mga Kristiyano).

un mujer que ta agarra largo bijuco el que ya puede sila mira na aplaya. Ya grita kanila el mujer "Bueno pa bira ya camo otravez y si jende tiene grande desgracia ay llega caniño".

(isang babaeng may dalang mahabang tungkod ang nakita nila sa dalampasigan.Sumigaw ang babae "bumalik na kayo sa iyong pinanggalingan at kung hindi may darating sa iyong na matinding kapahamakan”).

Ya salva el Bolong. Ta cre el maga gente que el mujer el La Virgen del Rosario. Desde aquel tiempo, el maga viviente de Bolong ta celebra el fiesta de La Virgen del Rosario todo el primer Domingo de Octubre.

(Nailigtas ang Bolong. Pinaniniwalaan ng mga tao na ang babae ay ang Birhen ng Rosario. Hanggang sa panahong ito, ipinagdiriwang ng mga tao sa Bolong ang pista ng Birhen ng Rosario tuwing unang linggo ng Oktubre).

Inilalarawan sa leyenda na gustong atakihin ng mga Moro ang mga Kristiyanong naninirahan sa isang baryo, subalit bago pa man sila makababa sa kanilang sinasakyang barko ay nagpakita na sa kanila ang isang magandang babae na pinaniniwalaan ng mga Kristiyanong ang Mahal na Birhen. At dahil natakot dito ang mga Moro hindi natuloy ang kanilang masamang balak kaya nakaligtas ang mga Kristiyano sa nasabing pag-atake. Ang Mahal na Birhen ay may napakalaking impluwensiya sa pananampalataya ng mga Kristiyanong Zamboangueño. Kinikilala siya bilang "Ina ng Panginoong Hesus", nagiging modelo siya sa kababaihan, nirerespeto at sinasampalataya. Ang kanyang pagtulong at pagpapakita ay hindi na rin bago sa mga tao sapagkat marami-rami na rin ang nagsalaysay ng kanilang mga karanasan sa milagro ng Mahal na Birhen at kahit hindi ito nakikita ng ibang tao ay naniniwala pa rin sila na totoo ito. Patunay na riyan ang napakaraming deboto ng Birhen del Pilar dito sa lungsod Zamboanga. Iba- iba man ang tawag sa kanya batay sa lugar kung saan siya nagpakita tulad ng barangay Bolong na kilala siya sa tawag na Nuestra Señora del Rosario, Mariam naman sa mga Muslim subalit iisa pa rin ang tinutukoy nito - ang Birheng Maria.

Ang Aksidente at Pagkamatay ni Graciano - Ipinakikita sa leyendang El Alma Agricido na ang aksidenteng naganap at pagkamatay ng katawang lupa ni Graciano ang salik na nakaimpluwensiya sa pananampalatayang Zamboangueño na ang kaluluwa ng namatay ay muling nabubuhay upang humarap sa Panginoon para sa paghuhukom.

Un dia, ya tiene accidente si Graciano y ya muri le. Pero no hay recibi su alma na cielo por causa del tonterias yah ace le con Isabel. Ya manda bira su alma na mundo na forma humano para puede le paga el tonterias ya hace le. Necesita le suporta con Isabel y su anak antes de recibi conele na cielo.

(Isang araw naaksidente si Graciano at siya'y namatay. Hindi tinanggap ang kanyang 
Mga leyendang Chabacano: Salamin sa pananampalatayang Zamboangueño

kaluluwa sa langit dahil sa kalokohang ginawa niya kay Isabel. Pinabalik ang kanyang kaluluwa sa mundo sa anyong tao upang pagbayaran ang kanyang panloloko. Kailangan niyang suportahan si Isabel at ang kanyang anak bago siya tanggapin sa langit). (Mula sa ikalawang talata ng El Alma Agricido)

Sa pagsasalaysay sa una at ikalawang pangungusap ng talatang sinipi sa itaas ay makikitang naaksidente ang isang tao kaya siya'y namatay. Sa pagtatapos ng ating makamundong paglalabay, ang kamatayan ay isang di matatakasang paghuhukom na magdadala sa tunay na kinalabasan ng buhay ng isang tao (cf. CCC 1021-22). Ito ay binanggit ni Hesus sa kanyang parabula tungkol sa kaawa-awang si Lazarus (cf. Lk 16:22) at ang kanyang pangako sa isang mabuting magnanakaw: "in this day you will be with me in paradise" (Lk 23:43). Itinuturo ng simbahan na ang mga namatay na may mabuting kalooban ay tinatanggap kaagad sa langit, at makikita at makikilala nila ang Panginoon mismo bilang una hanggang ikatlong persona, habang ang mga namatay na may kasalanang mortal ay bababa sa impiyerno (Counsel of Florence, ND 2309).

Sa pagpapatuloy ng kuwento sa leyendang nabanggit pagkatapos mamatay ng tao dulot ng aksidente ay hindi tinanggap ang kanyang kaluluwa sa langit dahil may ginawa siyang kalokohan sa isang babae. Naniniwala ang mga Zamboangueño na kapag namatay ang isang tao ay katawan lamang niya ang mawawala subalit ang kanyang kaluluwa ay mabubuhay at haharap sa Panginoon para sa paghuhukom. Ito ay tinatawag na resureksiyon.

Sa mga nailahad sa itaas, ipinakikita na ang bawat paraan ng pananampalataya ng mga Zamboangueño ay may mga salik na nakaimpluwensiya dito. Ang mga salik pangkalikasan na batay sa natural na mga pangyayari o penomena, at mga salik pantao na batay sa impluwensiya ng mga kapaligirang panlipunan, pang-institusyon, pangkultura at penomenang panlipunan na gawa mismo ng mga tao sa lipunan. Bawat pangyayari sa buhay ng mga Zamboangueño na inilahad sa mga leyenda ay tinatapatan nila ng pananampalataya sa Panginoon.

\section{Kongklusyon}

Layunin ng pag-aaral na ito na tukuyin ang iba't ibang salik na nakaimpluwensiya sa pananampalatayang zamboangueňo na makikita sa mga leyendang Chabacano ng lungsod Zamboanga. Ginamit ang mga teoryang historikal at sosyolohikal sa pagtukoy sa mga nabanggit na salik.

Mula sa pagsusuring ginawa sa labinlimang (15) mga leyendang Chabacano, may sampung paraan ng pananampalataya ang mga Zamboangueño na inilahad sa mga leyendang binanggit, ito ay ang Pagdarasal (reza), Pagpruprusisyon (procession), Pagbabasa ng Bibliya (liende Biblia), Pagbibinyag (Bautismo), Paniniwala sa Panginoon, (Ta cre con el Dios), Pagdiriwang ng Pista (Celebra fiesta), Pagtatayo ng Kapilya (Hace Capilla/Ermita), Pagnonobena, pagrorosaryo at pagmimisa (Novena, Rosario, Misa), Paniniwala sa muling pagkabuhay ng kaluluwa (resureccion del Alma), Pangungumpisal (Compesa). Bawat paraan ng pananampalataya ay may salik na nakaimpluwensiya rito na maaaring puwersang pangkalikasan tulad ng pagkahulog ng bunga ng niyog sa harap ng pusa, paglaganap ng kolera sa baryo at pagkaligtas ng mga tao laban sa naturang sakit, pagkamatay ng mga pananim dahil sa tagtuyot, taggutom sa baryo, pagkakasakit at pagkamatay ng mga tao dahil sa kolera, at pagdadakila sa birhen, paglitaw ng imahen ng santo sa tabla, pagkakaroon ng matinding sakit ng babae at pagkamatay. Ang mga salik namang nakaimpluwensiyang batay sa puwersang pantao ay ang paglusob ng mga bandidong grupo, pagkakaroon ng isang relihiyosong pagdiriwang, pagiging relihiyoso ng mga Moro, pagdiriwang ang unang sentinaryo ng baryo Tetuan, pagdiriwang sa araw ng Patron ng baryo, pananampalataya sa santo, pagkakaroon ng matalik na kaibigang Katoliko ng prinsesa na siyang naging daan sa pagbabago ng kanyang pananampalataya, pagkaligtas ng baryo laban sa mga sumalakay na Moro, at ang aksidente at pagkamatay ng katawang lupa.

Batay sa natuklasan sa pagsusuri sa mga datos, nabuo ang konklusyong na malaki ang papel na ginampanan ng mga historikal na kaganapan at mga institusyong tulad ng pamilya, simbahan, edukasyon, batas, politika, at kultura sa paghubog ng pananampalataya ng mga Zamboangueño, kalakip na ito sa lahat ng siklo ng kanilang 
Reyes, A.

buhay at pamumuhay mula pagkasilang hanggang kamatayan. Ang mga nakalap na mga salik na siyang nakaimpluwnsiya sa paraan ng pananampalataya ng mga Zamboangueño batay sa mga leyendang Chabacano ng lungsod Zamboanga ay nagsisilbing repleksiyon sa uri ng paniniwala at kaugalian batay sa espiritwal na pananaw sa buhay o pananampalataya ng mga Zamboangueño. Bagama't ang mga Zamboangueño ay maaaring Kristiyano (katoliko o di- katoliko) at Muslim (Islam), sila ay parehong nagpapakita ng pagkakaroon ng matinding pananampalataya sa Panginoon. Malaki ang naging impluwensiya ng mga dayuhang mananakop partikular na ang mga Kastila sa kanilang pagpapalaganap ng Kristiyanismo sa mga Kristiyanong Zamboangueño at ang impluwensiya ng Islam na pamana naman ng mga Arabe sa mga Muslim ng Mindanao sa pagkakaroon ng matinding pananampalataya ng mga Zamboangueño.

\subsection{Rekomendasyon}

Batay sa kinalabasan ng pag-aaral, iminumungkahi ng mananaliksik para sa mga mag-aaral, guro at mga mananaliksik sa hinaharap na magsagawa ng katulad na pag-aaral gamit ang ibang genre ng oral na literatura na susuriin upang magkaroon nang mas malalim na pagkilala sa mga Zamboangueño.

Magsagawa din ng katulad na pag-aaral sa iba pang tribo at gamiting hambingan ang pag-aaral na ito upang malaman ang iba pang salik sa pananampalataya ng ibang liping Pilipino.

Iminumungkahi rin sa lokal na pamahalaan ng lungsod kasama na ang mga simbahan na magkaroon ng mga proyektong lalong magpapayaman at magpapanatili sa ganitong uri ng kultura lalo na sa mga susunod pang henerasyon.

\section{Mga Sanggunian}

Catholic Bishops' Conference of the Philippines [CBCP]. (1997). Cathechism for Filipino Catholics. ECCE.

Cuartocruz, O. B. (1995). Zamboanga Chabacano folk literature. Tokyo.

del Castillo, F (2015). Christianization of the Philippines: Revisiting the contributions of baroque churches and religious arts. Mission Studies, 31(1), 47-65. https://doi.org/10.1163/15733831-12341379

del Castillo, F. (2016). Gospel-culture relationship of traditional Filipino religion and Catholicism. The International Journal of Religion and Spirituality in Society, 6(2), 41-47. https://doi.org/10.18848/2154-8633/CGP/v06i02/51010

Fuellenbach, J. (1992). Proclaiming his kingdom: Meditations for personal recollection. Logos Publication.

Julian, A. B., \& Lontoc, N. S. (2016). New journey of the Filipino race [Bagong lakbay ng lahing Pilipino]. Phoenix Publishing House.

Semorlan, T. P. (1984). Chabacano legends [Mga leyendang Chabacano]. MSU-IIT Coordination Center for Research and Development.

Yap-Aizon, J. G., Potenciano, C. R., Jamiro, S. M. A., \& Mangaser, V. D. (2016). Riquezas: Chavacano essays and studies. Local government of Zamboanga. 